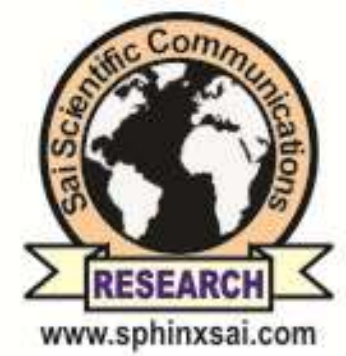

Phardinters

International Journal of PharmTech Research CODEN (USA): IJPRIF, ISSN: 0974-4304, ISSN(Online): 2455-9563

Vol.10, No.4, pp 280-286,

2017

\title{
Traditional Risk Factors as Determinants of Heart Rate Recovery in Patients with Diabetes Mellitus type 2 Without Known Coronary Artery Disease
}

\author{
Sanny Silaban ${ }^{1,2 \star}$, A Afif Siregar ${ }^{1,2}$, H Hasan $^{1,2}$ \\ ${ }^{1}$ Departement of Cardiology and Vascular Medicine, Medical Faculty, University of \\ Sumatera Utara, JI. Dr.T.Mansur No.5.Kampus USU,Medan 20155, Indonesia \\ ${ }^{2}$ Haji Adam Malik General Hospital, JI Bunga Lau No.17, Kemenangan Tani, Medan \\ 20136, Indonesia
}

\begin{abstract}
The impact of Traditional risk factors on heart rate recovery (HRR) has not been studied in patients Diabetes Mellitus type 2 without known coronary artery disease (CAD). For this reason, we sought to determine the association between HRR as cardiac autonomic dysfunction marker and traditional risk factors. The study was conducted with a crosssectional study involving 89 patients with Type 2 Diabetes Mellitus without known having coronary artery disease. The data was taken through anamnese and laboratory tests, and subjects who met the criteria, were tested for a treadmill exercise to assess heart rate recovery in the first minute. In bivariate analysis Dyslipidemia, Hypertension, smoker, age, duration of $\mathrm{DM} \geq 5$ years, $\mathrm{HbA1C} \geq 7.5$, Peak Heart rate, functional capacity and ST depression ischemic have an association with heart rate recovery. In multivariate analysis patients with hypertriglyceride, smoker, overweight, duration of diabetes $\geq 5$ years and $\mathrm{HbAlC} \geq 7,5$ have lower heart rate recovery significantly. Traditional risk factors are determinant factors for heart rate recovery in patients with Diabetes Mellitus type 2 without known coronary artery disease.

Keywords : Exercise test, Cardiac disease prevention, Cardiac autonomic dysfunction.
\end{abstract}

\section{Introduction}

Diabetes Mellitus is an important and independent risk factor for cardiovascular mortality and morbidity ${ }^{1}$. Most patients with DM develop autonomic neuropathy, which is one of the more common complications in this population. Fifteen studies that use different end points reported prevalence rates of $1 \%$ to $90 \%$ for its insidence ${ }^{2}$. Damage to autonomic nerve fibers that innervate the heart and blood vessels consequently lead to abnormalities in heart rate (HR) control and vascular dynamics ${ }^{3}$. Comparatively, the mortality of DM patients manifestingan autonomic neuropathy is markedly higher than DM patients without autonomic neuropathy ${ }^{4,5}$.

Abnormal heart rate recovery (HRR) after exercise is an easy to measure marker of reduced parasympathetic activity. It has been found to be an independent predictor of mortality in submaximal as well as

International Journal of PharmTech Research, Vol.10, No.4, pp 280-286, (2017)

http://dx.doi.org/10.20902/IJPTR.2017.10435 
in symptom-limited exercise stress testing ${ }^{6,7}$. HRR is defined as the reduction in heart rate from the peak of strenuous exercise to 1 minute after termination of exercise. It is a new index clinically significant in evaluating the cardiac autonomic system, especially the parasympathetic nervous function after exercise. Panzer et al reported that abnormal HRR was present among $42 \%$ of patients with impaired fasting glucose and 50\% with diabetes $^{8}$. HRR is considered as a strong predictor of increased cardiovascular disease and overall mortality, independently of other cardiac risk factors ${ }^{9}$. Several studies have also shown that abnormal HRR is a predictor of poor prognosis in patients with heart failure ${ }^{10,11}$. In addition, abnormal HRR is associated with severity of coronary artery disease $\mathrm{e}^{12}$.

However, patients with Type 2 Diabetes Mellitus (T2DM) have typically many shared complications, e.g., obesity and hyperlipidemia, which can be considered tobe risk factors of T2DM rather than its consequences and which are known to affect in HRR in non-diabetic population ${ }^{13,14}$. Also reduced physical activity level and low physical fitness, which is associated with blunted HRR in a healthy population are common among patients with T2DM ${ }^{13,15,16}$. We, therefore hypothesized that HRR of T2DM patientsmight not be altered only because of the disease itself or its relatedcomorbidities but also because of other earlier risk factors, e.g., obesity,hypertension,hyperlipidemia and lifestyle etc smoker, age, with low physical activity.In the present study, we examined the association between cardiovascularautonomic function as assessed by HR recovery after exerciseand other potential factors, including demographic characteristics, metabolic variables that we conclude as traditional risks for coronary disease among patients with T2DM with unknown coronary artery disease $(\mathrm{CAD})$.

\section{Materials and Methods}

The population of the present study consisted of 89 consecutive participants with T2DM who underwent treadmill exercise with Bruce protocol. The subjects for the study were male 67 (75\%), and female $22(25 \%)$, meanage 55 $\pm 7,5$ year, were volunteers for a routine health check-upon the Cardiac Centre of Haji Adam Malik Hospital, Medan, Indonesia. The subject's past medical histories were obtainedthrough an anamnesis. Those who had history of stroke, disability, COPD, established heart disease, and the other chronic heart disease were exlcluded. Subjects withhypertensiveor on antihypertensive medication except for beta blocker dan Calcium channel blockers (CCB) non dyhidropiridine (DHP)were includedin the study. We obtained written consents from all subjects, and the study was approved by Adam Malik Hospital and University of Sumatera Utara Medical School Institutional Review Board.

\section{Demographic and Laboratory tests}

Blood sampling for serum lipid profile and glucose wasobtained after at least $14 \mathrm{hr}$ of fasting. Post prandial blood glucose obtained after 2 hours meal. Diabetes Mellitus (symptom of diabetes and plasma glucose concentration $\geq 200 \mathrm{mg} / \mathrm{dl}$ or fasting plasma glucose $\geq 126 \mathrm{mg} / \mathrm{dl}$ or $2 \mathrm{hp}>200 \mathrm{mg} / \mathrm{dl}$ ) was diagnosed based on laboratory, patients information and medical history. Traditional risk defines as dyslipidemia, smoker, age $>55$ years, hypertension and obesity. As for Diabetes status, we include blod glucose control (HbA1C) and time duration the subject established with T2DM. Subjects were defined by their smoking habits as current or nonsmokers. Ex-smokers were considered those who quit smoking for at least 3 years. Body weight and height were measured in light clothing, and body mass index (BMI) was calculated. Hypertension was defined according to the current guidelines ${ }^{17}$.

\section{Exercise Protocol}

Treadmill exercise stress test was done in all subjects using the Bruce protocol. Blood pressure, HR and the Borg scale of rating of perceived exertion (RPE) 20 weremeasured at 2 minute of each stage of the exercise ${ }^{17}$.The exercise wasstopped when the subject demanded cessation of the treadmilldue to exhaustion, or if the heart rate achieved was morethan $85 \%$ of estimated maximal HR (220-age), or if the RPEwas more than 18. During the recovery phase, the subjects continued to walk for 30 second at the speed of $1.2 \mathrm{mph}$ and then they satdown for 5 min with continued medical monitoring. HRR was calculated as the decrease of HR per minutebetween the peak exercise period and $1 \mathrm{~min}$ post exercise. Abnormal HRR define as the reduction of heart rate $\leq 12$ b.p.m from peak heart rate to 1 minute after the termination of exercise. Those subjects who showed malignant arrhythmia, severe hypertension or hypotension, or unfinished exercise due to neuromuscular problem during exercise were excluded from this study. Positive myocardial ischemia was defined as horizontal or downsloping ST segment depression of $\geq 0.1 \mathrm{mV}$ for $80 \mathrm{~ms}$ in 3 consecutive beats. 


\section{Statistical Analysis}

Data with normal distribution expressed as a mean \pm standard deviation and the others expressed as a median (lower-upper) value. Comparisons of means between the two groups were made by usinga Student ttest. Bivariate analysis between variables was evaluated by Chi square. Regression logisticmodel was constructed with HRR as the dependent variable, and independent variables were those variables showingsignificant association with HR recovery upon uni and bivariate analysis. Back stepwise regression was used for the final model selection. Criteriafor stepwise regression was 0.25 to included in multivariate analysis. Association between the independentvariables was explored and insignificant associationterms were removed from the final model. A $p$ value $<0.05$ was considered statistically significant. SPSS 18 for was used for the analysis.

\section{Result}

Clinical Characteristic Demographic and laboratory characteristics of the population are shown in Table 1. The population study was around 55 $\pm 7,39$ years old. Half of the subjectshad a sedentary lifestyle, with only $50,2 \%$ doing regular exercisemore than three times per week.Fifty-eight percent of the subjectsreported that they were either hypertensive or on antihypertensivemedications. Fifty-eight point one percent were smokers, and $63 \%$ subjects have dyslipidemia. There is significant different value in Lipid profile (HDL-c, LDL-c, TG) between subject with abnormal HRR $(\leq 12$ b.p.m) and normal HRR ( $>12$ b.p.m). In Diabetic profile, there are significant different value in HbA1C, fasting blood glucose and Duration of T2DM between normal and abnormal HRR. The BMI also show a significant between both of the group $27(12,8-35)$ VS 24 (19-29),$p=$ 0,001. As is shown in Table 2, these Treadmill test variables displaya significant difference between those with abnormal and normal HRR proportion of peak heart rate, Mets, Chronotropic Incompetence, were different between the two groups.

Table 1. Demographic Characteristic Patients

\begin{tabular}{|c|c|c|c|c|}
\hline \multirow[t]{2}{*}{ Characteristic } & \multicolumn{2}{|c|}{ Heart Rate Recovery } & \multirow[t]{2}{*}{ All patients } & \multirow[t]{2}{*}{ P Value } \\
\hline & $\begin{array}{c}\leq 12 \text { b.p.m } \\
\mathrm{N}=46\end{array}$ & $\begin{array}{c}>12 \text { b.p.m } \\
\mathrm{N}=43\end{array}$ & & \\
\hline Age (median), years & $57 \pm 6,5$ & $52 \pm 7,39$ & $55 \pm 7,5$ & 0,001 \\
\hline Male & $34(38 \%)$ & $33(37,5 \%)$ & $67(75 \%)$ & 0,760 \\
\hline Hypertension & $35(38 \%)$ & $17(19 \%)$ & $52(58 \%)$ & 0,001 \\
\hline Smoker & $27(30 \%)$ & $11(28,9 \%)$ & $38(58,9 \%)$ & 0,003 \\
\hline Dyslipidemia & $35(38 \%)$ & $23(25 \%)$ & $58(63 \%)$ & 0,01 \\
\hline HDL-c (mg/dl) & $38,6 \pm 4,7$ & $48 \pm 7,42$ & $43 \pm 6,9$ & 0,003 \\
\hline LDL-c (mg/dl) & $178(92-265)$ & $132(90-211)$ & $156(90-265)$ & 0,045 \\
\hline $\mathrm{TG}(\mathrm{mg} / \mathrm{dl})$ & $197 \pm 51$ & $141 \pm 46$ & $170 \pm 56$ & 0,031 \\
\hline Total Cholesterol & $241(156-376)$ & $196(167-319)$ & $212(156-376)$ & 0,076 \\
\hline $\mathrm{HbA1C}$ & $8,2(6,4-11,6)$ & $6,5(6-10,2)$ & $7,5(6-11,6)$ & 0,001 \\
\hline Duration of T2DM & $6(3-10)$ & $4(1-7)$ & $4(1-10)$ & 0,034 \\
\hline FPG (mg/dl) & $147 \pm 46$ & $119 \pm 28$ & $135 \pm 51$ & 0,02 \\
\hline 2h post prandial glucose $(\mathrm{mg} / \mathrm{dl})$ & $212(133-332)$ & $178(121-245)$ & $210 \pm 51,9$ & 0,001 \\
\hline$\overline{B S A}$ & $<1,8(1,4-2,7)$ & $1,8(1,4-2,1)$ & $1,8(1,4-2,7)$ & 0,275 \\
\hline$\overline{\text { BMI }}$ & $27(21,8-35)$ & $24(19-29)$ & $26(19-35)$ & 0,001 \\
\hline $\mathrm{CCB}$ & $22(24 \%)$ & $5(5 \%)$ & $27(29 \%)$ & 0,07 \\
\hline$\overline{\text { ACE-I/ARB }}$ & $28(29,2 \%)$ & $11(12 \%)$ & $39(41,2) \%$ & 0,45 \\
\hline T2DM therapy & $46(51 \%)$ & $43(49 \%)$ & $89(100 \%)$ & \\
\hline Oral & $34(38 \%)$ & $28(31 \%)$ & $62(78 \%)$ & 0,48 \\
\hline Insulin & $12(13 \%)$ & $15(16 \%)$ & $27(22 \%)$ & 0,373 \\
\hline Lipid Lowering Drug & $21(23 \%)$ & $29(32 \%)$ & $50(55 \%)$ & 0,054 \\
\hline Exercise & $17(19 \%)$ & $28(31,2 \%)$ & $45(50,2 \%)$ & 0.011 \\
\hline
\end{tabular}

FPG: Fasting Plasma Glucose; BMI: Body Mass Index; TG: Triglycerides; HDL-c: High-Density Lipoprotein cholesterol; LDL-c: Low-Density lipoprotein Cholesterol; BMI: Body Mass Index; BSA: Body Surface Area; CCB: Calcium Chanel Blocker; ACE-I: Angiotensin Converting Enzym Inhibitor;ARB: Angiotensin II Receptor Blocker. 
Table 2. Characteristic Treadmill Test Result

\begin{tabular}{|l|l|l|l|l|}
\hline \multirow{2}{*}{ Variable } & \multicolumn{2}{|c|}{ Heart Rate Recovery } & \multirow{2}{*}{ All patient } & \multirow{2}{*}{ P Value } \\
\cline { 2 - 3 } & \multicolumn{1}{|c|}{$\leq 12$ b.p.m } & \multicolumn{1}{|c|}{$>$ b.p.m } & & \\
\hline Peak Heart Rate & $136(76-187)$ & $156(111-178)$ & $148(76-178)$ & 0,001 \\
\hline Peak Sistolic BP $(\mathrm{mmHg})$ & $179,2 \pm 28,11$ & $179,6 \pm 21,34$ & $179 \pm 24,9$ & 0,92 \\
\hline Peak Diastolic BP $(\mathrm{mmHg})$ & $79(58-126)$ & $77(65-118)$ & $77(58-126)$ & 0,86 \\
\hline Mets & $7(1,8-10,2)$ & $9,2(5-11)$ & $7,6(1,8-11)$ & 0,001 \\
\hline $\begin{array}{l}\text { Duration Treadmil test } \\
\text { (minute) }\end{array}$ & $5,09(2,4-9,5)$ & $6,57(3,4-10,49)$ & $6,4(2,4-10,49)$ & 0,002 \\
\hline Chronotropic Incompetence & $25(28 \%)$ & $7(7 \%)$ & $32(35 \%)$ & 0,001 \\
\hline ST depression positive & $21(23 \%)$ & $5(5,6 \%)$ & $26(29 \%)$ & 0,001 \\
\hline
\end{tabular}

BP: Blood Pressure; Mets: Metabolic Equivalents

Table 3. Bivariate Analyses between HRR and Traditional Risk Factors

\begin{tabular}{|c|c|c|c|c|c|c|}
\hline \multirow[t]{2}{*}{ Variable } & \multicolumn{2}{|c|}{ Heart Rate Recovery } & \multirow[t]{2}{*}{$\mathbf{P}$} & \multirow[t]{2}{*}{ OR } & \multicolumn{2}{|c|}{ CI } \\
\hline & $\leq 12$ b.p.m & $>12$ b.p.m & & & Min & Max \\
\hline $\begin{array}{r}\text { Age(years) } \\
\bullet \quad \geq 55 \\
\bullet \quad<55\end{array}$ & $\begin{array}{l}28(68,3 \%) \\
18(37,5 \%)\end{array}$ & $\begin{array}{l}13(31,7 \%) \\
30(62,5 \%)\end{array}$ & 0,04 & $\mathbf{3 , 5 9}$ & 1,48 & 8,54 \\
\hline $\begin{array}{cl}\text { Gender } & \\
\bullet & \text { Male } \\
\bullet & \text { Female } \\
\end{array}$ & $\begin{array}{l}34(50,7 \%) \\
12(54,5 \%) \\
\end{array}$ & $\begin{array}{l}33(49,3 \%) \\
10(45,4 \%) \\
\end{array}$ & 0,76 & 0,89 & 0,32 & 2,25 \\
\hline $\begin{array}{c}\text { Hypertension } \\
\bullet \quad \text { Yes } \\
\text { - No } \\
\end{array}$ & $\begin{array}{l}35(67,3 \%) \\
11(29,7 \%)\end{array}$ & $\begin{array}{l}17(32,7 \%) \\
26(70,3 \%)\end{array}$ & 0,001 & $\mathbf{4 , 8 1}$ & 1,954 & 12,12 \\
\hline $\begin{array}{cc}\text { Smoker } \\
\bullet \quad \text { Yes } \\
\bullet \quad \text { No } \\
\end{array}$ & $\begin{array}{l}27(71,1 \%) \\
19(37,3 \%) \\
\end{array}$ & $\begin{array}{l}11(28,9 \%) \\
32(62,7 \%) \\
\end{array}$ & 0,003 & 4,13 & 1,667 & 10,19 \\
\hline $\begin{array}{c}\text { Dyslipidemia } \\
\bullet \quad \text { Yes } \\
\bullet \quad \text { No } \\
\end{array}$ & $\begin{array}{l}41(59,4 \%) \\
5(25 \%) \\
\end{array}$ & $\begin{array}{l}28(40,6 \%) \\
15(75 \%) \\
\end{array}$ & 0,001 & 4,76 & 1,12 & 6,8 \\
\hline $\begin{aligned} & \text { Lipid } \text { Profile } \\
& \text { - } \text { HDL-c } \leq 40 \\
& \text { - } \text { HDL-c }>40 \\
&\end{aligned}$ & $\begin{array}{l}22(75,9 \%) \\
24(40 \%)\end{array}$ & $\begin{array}{l}7(24,1 \%) \\
36(60 \%) \\
\end{array}$ & 0,02 & 4,71 & 1,74 & 12,7 \\
\hline $\begin{array}{ll}- & \text { LDL-c } \geq 130 \\
- & \text { LDL-c }<130\end{array}$ & $\begin{array}{l}34(60,7 \%) \\
12(36,4 \%)\end{array}$ & $\begin{array}{l}22(39,3 \%) \\
21(63,6 \%)\end{array}$ & 0,03 & 2,7 & 1,12 & 6,58 \\
\hline $\begin{array}{ll}-\mathrm{TG} \geq 150 \\
\text { - } & \mathrm{TG}<150 \\
\end{array}$ & $\begin{array}{l}36(80 \%) \\
10(22,7 \%) \\
\end{array}$ & $\begin{array}{l}9(20 \%) \\
34(77,3 \%) \\
\end{array}$ & 0,001 & 8,31 & 3,13 & 21,6 \\
\hline $\begin{array}{ll}- & \text { Total Cholesterol } \\
& \geq 200 \\
\text { - } & \text { Total Cholesterol } \\
& <200\end{array}$ & $\begin{array}{l}34(68,8 \%) \\
12(30,8 \%)\end{array}$ & $\begin{array}{l}16(32 \%) \\
37(69,2 \%)\end{array}$ & 0,04 & 4,78 & 1,93 & 11,7 \\
\hline $\begin{aligned} \text { BMI } & \\
\bullet & \geq 25 \\
\text { - } & <25\end{aligned}$ & $\begin{array}{l}32(68,1 \%) \\
14(33,3 \%)\end{array}$ & $\begin{array}{l}15(31,9 \%) \\
28(66,7 \%)\end{array}$ & 0,03 & 4,24 & 1,35 & 8,01 \\
\hline
\end{tabular}

BMI: Body Mass Index; TG: Trygliceride; HDL: High-Density Lipoprotein cholesterol; LDL-c: Low-Density lipoprotein Cholesterol; BMI: Body Mass Index 
Table 4. Bivariate Analyses between HRR and T2DM status

\begin{tabular}{|c|c|c|c|c|c|c|}
\hline \multirow[t]{2}{*}{ Variable } & \multicolumn{2}{|c|}{ Heart Rate Recovery } & \multirow[t]{2}{*}{$\mathbf{P}$} & \multirow[t]{2}{*}{ OR } & \multicolumn{2}{|c|}{ CI } \\
\hline & $\leq 12$ b.p.m & $>12$ b.p.m & & & Min & Max \\
\hline $\begin{array}{r}\text { HbA1C } \\
\cdot \quad \geq 7,5 \\
\bullet \quad<7,5\end{array}$ & $\begin{array}{l}33(80,5 \%) \\
13(26,5 \%)\end{array}$ & $\begin{array}{l}17(19,5 \%) \\
26(73,5 \%)\end{array}$ & 0.005 & 13,1 & 4,6 & 36,4 \\
\hline $\begin{aligned} \text { FPG } & \\
\bullet & \geq 126 \\
\bullet & <126\end{aligned}$ & $\begin{array}{l}12(26,6 \%) \\
34(77,3 \%)\end{array}$ & $\begin{array}{l}33(73,3 \%) \\
10(22,7 \%)\end{array}$ & 0,06 & 4,01 & 0,27 & 2,87 \\
\hline $\begin{array}{c}\text { 2h post prandial } \\
\bullet \quad \geq 200 \\
\bullet \quad<200\end{array}$ & $\begin{array}{l}28(70 \%) \\
18(36,7 \%)\end{array}$ & $\begin{array}{l}12(30 \%) \\
31(63,3 \%)\end{array}$ & 0,056 & 1,25 & 0,82 & 4,56 \\
\hline $\begin{array}{r}\text { Duration T2DM } \\
\bullet \quad \geq 5 \text { years } \\
\bullet \quad<5 \text { years }\end{array}$ & $\begin{array}{l}26(78 \%) \\
20(35,7 \%)\end{array}$ & $\begin{array}{l}7(22 \%) \\
36(64,3 \%)\end{array}$ & 0,012 & 6,86 & 2,46 & 18,3 \\
\hline
\end{tabular}

FPG: Fasting Plasma Glucose; T2DM: Type 2 Diabetes Mellitus

Table 5. Logistic Regression with HRR as Dependent Variable

\begin{tabular}{|l|l|l|l|l|}
\hline Variable & Coef & P & OR & CI 95\% \\
\hline Smoker & 0,801 & 0,042 & 2,21 & $1,312-15,296$ \\
Triglyserides & 1,27 & 0,015 & 3,567 & $1,378-20,23$ \\
BMI & 0,871 & 0,024 & 2,388 & $2,594-23,31$ \\
Duration of T2DM & 2,451 & 0,003 & 11,09 & $2,402-53,59$ \\
HBA1C & 2,381 & 0,024 & 10,08 & $1,447-71,72$ \\
Peak Heart Rate & $-1,574$ & 0,015 & 0,265 & $0,006-0,913$ \\
ST Depression positive & 1,412 & 0,184 & 2,21 & $1,83-3,813$ \\
ischemia & & & & \\
\hline
\end{tabular}

In Bivariat analysis as in table 3 and 4, subjects who older than 55 years old [OR 4,81 (1,95-12,12)], hypertension [OR 4,81(1,954-12,12)], smoker[OR 4,13(1,667-10,19)], dyslipidemia [OR 4,76(1,12-6,8)], HDLc $<40$ [OR 4,71(1,74-12,73)], LDL-c $\geq 130$ [OR 2,7(1,12-6,58) ], TG $\geq 150$ [OR 8,31(3,13-21,60)], BMI $\geq 25$ [OR 4,24(1,35-8,01)] have higher probability to get abnormal heart rate recvovery. HbA1C $\geq 7,5$ has higher probability to abnormal heart rate recovery [OR $1,31(4,6-36,4)], p=0,005$ as seen in duration of diabetes $\geq 5$ years $[\mathrm{OR} 6,86(2,46-18,30)], p=0,012$.

By multivariate analysis in table 5 , the presence of Triglyceride $\geq 150$, smoker, $\mathrm{BMI} \geq 25, \mathrm{HbA1C} \geq 7.5$, Duration of $\mathrm{DM} \geq 5$ years were independently associated with slower HRR after an adjustment forthe other variables. Peak heart rate and ischemic response in treadmill test also give a significant association with delay of HRR.

\section{Discussion}

As shown in table 5, Triglyseride, BMI, Smoker, Duration of T2DM, HbA1C have independent association with the abnormality of heart rate recovery as a marker of subclinical cardiac diabetic neuropathy. Peak heart rate during exercise, and positive ST depression in precordial lead also showed significant association. This result shows that traditional risk factors can determine abnormal heart rate recovery as seen in coronary artery disease. Christina et al in 2001 also showed a similiar result with heart rate variability as its cardiac neuropathy marker. In a cross sectional study covered by $600 \mathrm{DM}$ patients, the regresion analysis showed cardiac otonomic neuropaty incidence increase with positif corelation with hypertension, smoker, duration of diabetes, LDL-c, Cholesterol triglyseride and HbA1C. HbA1C value in this study was $8 \pm 1,8$ with $p<0,05^{18}$. EURODIAB study also showed that there is a correlation between cardiac autonomic dysfunction with duration of DM (cut of point 8 years), smoker and HDL-c even though we have a different marker tool to established cardiac autonomic neuropathy ${ }^{19}$. It is necessary, however, to bear in mind that their patients had 
relatively well controlled glycemic state and that only a few of them had abnormal HR recovery according to the criteria mentioned.

However our limitation is the blood sample is taken only once to evaluate associaton between lipid profile and blood glucose status with heart rate recovery. Only one prior study has prospectively studied the relationship of slower HRR with change in risk factor levels over time, and similarly found no association between slower HRR and an increase in levels of glucose and insulin over time ${ }^{20}$.

\section{Conclusion}

In conclusion, traditional risk factor is significantly associated with impaired vagal reactivation as shown in slower heart rate recovery. Therefore, evaluation of all cardiovascular traditional risk factors is necesarry to prevent cardiac autonomic dysfunction in Diabetes Mellitus patients.

\section{References}

1. M. Wei, S.P. Gaskill, S.M. Haffner and M.P. Stern, Diabetes Care, 21, 1167 (1998); doi:10.2337/diacare.21.7.1167.

2. A.I. Vinik, R.E. Maser, B.D. Mitchell and R. Freeman, Diabetes Care, 26, 1553 (2003); doi:10.2337/diacare.26.5.1553.

3. M.P. Schumer and M.A. Pfeifer, Diabetes Spectr., 11, 227 (1998).

4. D.J. Ewing, D.Q. Borsey, F. Bellavere and B.F. Clarke, Diabetologia, 21, 18 (1981); doi:10.1007/BF03216217.

5. A. Moţăţăianu, R. Bălaşa, S. Voidăzan and Z. Bajko, Bio. Med Res. Int., •••, 507216 (2013).

6. C.R. Cole, J.M. Foody, E.H. Blackstone and M.S. Lauer, Ann. Intern. Med., 132, 552 (2000); doi:10.7326/0003-4819-132-7-200004040-00007.

7. E.O. Nishime, C.R. Cole, E.H. Blackstone, F.J. Pashkow and M.S. Lauer, JAMA, 284, 1392 (2000); doi:10.1001/jama.284.11.1392.

8. C. Panzer, M.S. Lauer, A. Brieke, E. Blackstone and B. Hoogwerf, Diabetes, 51, 803 (2002); doi:10.2337/diabetes.51.3.803.

9. A. Morshedi-Meibodi, M.G. Larson, D. Levy, C.J. O’Donnell and R.S. Vasan, Am. J. Cardiol., 90, 848 (2002); doi:10.1016/S0002-9149(02)02706-6.

10. M. Guazzi, J. Myers, M.A. Peberdy, D. Bensimhon, P. Chase and R. Arena, Int. J. Cardiol., 144, 121 (2010); doi:10.1016/j.ijcard.2008.12.149.

11. D.E. Thomas, S.A. Exton and Z.R. Yousef, Heart, 96, 1385 (2010); doi:10.1136/hrt.2009.188540.

12. D.P. Vivekananthan, E.H. Blackstone, C.E. Pothier and M.S. Lauer, J. Am. Coll. Cardiol., 42, 831 (2003); doi:10.1016/S0735-1097(03)00833-7.

13. P. Georgoulias, A. Orfanakis, N. Demakopoulos, P. Xaplanteris, G. Mortzos, P. Vardas and N. Karkavitsas, J. Nucl. Cardiol., 10, 498 (2003); doi:10.1016/S1071-3581(03)00530-0.

14. T. Kimura, T. Matsumoto, M. Akiyoshi, Y. Owa, N. Miyasaka, T. Aso and T. Moritani, Eur. J. Appl. Physiol., 97, 542 (2006); doi:10.1007/s00421-006-0207-8.

15. M.R. Carnethon, D.R. Jacobs, S. Sidney and K. Liu, Diabetes Care, 26, 3035 (2003); doi:10.2337/diacare.26.11.3035.

16. M.R. Carnethon, D.R. Jacobs Jr., S. Sidney, B. Sternfeld, S.S. Gidding, C. Shoushtari and K. Liu, Med. Sci. Sports Exerc., 37, 606 (2005); doi:10.1249/01.MSS.0000158190.56061.32.

17. E. Lurbe, R. Cifkova, J.K. Cruickshank, M.J. Dillon, I. Ferreira, C. Invitti, T. Kuznetsova, S. Laurent, G. Mancia, F. Morales-Olivas, W. Rascher, J. Redon, F. Schaefer, T. Seeman, G. Stergiou, E. Wühl and A. Zanchetti, J. Hypertens., 27, 1719 (2009); doi:10.1097/HJH.0b013e32832f4f6b. 
18. C. Voulgari, M. Psallas, A. Kokkinos, V. Argiana, N. Katsilambros and N. Tentolouris, J. Diabetes Complications, 25, 159 (2011); doi:10.1016/j.jdiacomp.2010.06.001.

19. P. Kempler, S. Tesfaye, N. Chaturvedi, L.K. Stevens, D.J. Webb, S. Eaton, Z. Kerényi, G. Tamás, J.D. Ward and J.H. Fuller, Diabet. Med., 19, 900 (2002); doi:10.1046/j.1464-5491.2002.00821.x.

20. M.R. Carnethon, D.R. Jacobs, S. Sidney and K. Liu, Diabetes Care, 26, 3035 (2003); doi:10.2337/diacare.26.11.3035. 